\title{
Single-Dish Radio Polarimetry in the F-GAMMA Program with the Effelsberg 100-m Radio Telescope
}

Tobias Beuchert ${ }^{1,2, a}$, Matthias Kadler ${ }^{2}$, Jörn Wilms ${ }^{1}$, Emmanouil Angelakis ${ }^{3}$, Lars Fuhrmann ${ }^{3}$, Ioannis Myserlis ${ }^{3}$, loannis Nestoras ${ }^{3}$, Alex Kraus ${ }^{3}$, Uwe Bach ${ }^{3}$, Eduardo Ros ${ }^{3,4,5}$, Christoph Grossberger ${ }^{1,2}$, and Robert Schulz ${ }^{1,2}$

${ }^{1}$ Remeis Sternwarte/ECAP, Bamberg, Germany

${ }^{2}$ Lehrstuhl für Astronomie, Würzburg, Germany

${ }^{3}$ Max Planck Institut für Radioastronomie, Bonn, Germany

${ }^{4}$ Astronomical Observatory, University of Valencia, Spain

${ }^{5}$ Dept. Astronomy, University of Valencia, Spain

\begin{abstract}
Studying the variability of polarized AGN jet emission in the radio band is crucial for understanding the dynamics of moving shocks as well as the structure of the underlying magnetic field. The 100-m Effelsberg Telescope is a high-quality instrument for studying the long-term variability of both total and polarized intensity as well as the electric-vector position angle. Since 2007, the F-GAMMA program has been monitoring the linear polarized emission of roughly 60 blazars at 11 frequencies between 2.7 and $43 \mathrm{GHz}$. Here, we describe the calibration of the polarimetric data at 5 and $10 \mathrm{GHz}$ and the resulting F-GAMMA full-Stokes light curves for the exemplary case of the radio galaxy $3 \mathrm{C} 111$.
\end{abstract}

\section{Introduction}

Incoherent synchrotron radiation of leptons accelerated in the jet's magnetic field of radio-loud Active Galactic Nuclei (AGN) [4] is the major emission mechanism in the radio band. Depending on the underlying field configuration and the orientation of the jet towards the line-of-sight a net degree of linear polarization of several percent for the core and shocked jet can be observed [9, 20]. Linear polarization studies allow us to probe both the underlying magnetic field and via variability studies also the evolution of shocked components [1,2].

Within the F-GAMMA program [3, 14] both total intensity (Stokes I) and linear polarimetric data of roughly 60 Fermi blazars as well as some radio galaxies have been observed monthly with the Effelsberg 100-m Telescope since 2007. Unlike the majority of the target sources, 3C 111 shows prominent extended emission, resembling a FR II morphology [5] with two radio lobes and hot spots. The extended radio emission exhibits a steep radio spectrum. Due to Faraday rotation we have to expect some degree of depolarization for integrated single-dish measurements towards lower radio frequencies [13]. High frequency observations, however, are sensitive to compact core- and jet emission.

The F-GAMMA program has produced an extensive data base for a large source sample at multiple wavelengths between 2.7 and $43 \mathrm{GHz}$. Here we describe the calibration of total intensity and linear polarization at 5 and $10 \mathrm{GHz}$. In particular we present results for the radio galaxy $3 \mathrm{C} 111$.

\section{Müller Calculus and Polarization Properties}

The so-called Müller Calculus [16] allows to calibrate both total intensity and polarimetric data. The polarization state of an electromagnetic wave can be described by the Stokes formalism with the Stokes four-vector $(I, Q, U, V)^{\mathrm{T}}$. From that the total intensity $I$, polarized intensity $P=\sqrt{Q^{2}+U^{2}+V^{2}}$, degree of polarization $P D=\sqrt{Q^{2}+U^{2}+V^{2}} / I$ and the electric vector position angle (EVPA) $\chi=0.5 \arctan U / Q$ can be derived. The aim is to compute the source rest-frame Stokes vector $\mathbf{S}_{\mathrm{src}}=(I, Q, U, V)_{\mathrm{src}}^{\mathrm{T}}$ from that at the observer's frame $\mathbf{S}_{\mathrm{obs}}=(I, Q, U, V)_{\mathrm{obs}}^{\mathrm{T}}$, which is affected by instrumental effects. The corresponding relation is

$$
\mathbf{S}_{\mathrm{obs}}=M_{i j} R_{i j} \mathbf{S}_{\mathrm{src}}
$$

The Müller matrix $M_{i j}$ contains all calibration factors, whereas $R_{i j}$ describes the rotation of the source rest-frame EVPA and thus the beam-integrated EVPA in the horizontal coordinate-system of the telescope with the parallactic angle. As incoherent synchrotron radiation is negligibly intrinsically circularly polarized, the Stokes $V$ parameter is negligible for most AGN jets [10, 13]. In turn we only calibrate for linear polarization here. Writing down the matrix elements yields

$$
\left(\begin{array}{l}
I \\
Q \\
U
\end{array}\right)_{\mathrm{obs}}=\left(\begin{array}{lll}
m_{1,1} & m_{1,2} & m_{1,3} \\
m_{2,1} & m_{2,2} & m_{2,3} \\
m_{3,1} & m_{3,2} & m_{3,3}
\end{array}\right)\left(\begin{array}{l}
I \\
Q \\
U
\end{array}\right)_{\mathrm{p}} .
$$

\footnotetext{
a e-mail: tobias.beuchert@ sternwarte.uni-erlangen.de
} 
Table 1. Channel allocation for the 5 and $10 \mathrm{GHz}$ receivers for both the main and weather horn $(\mathrm{M} / \mathrm{W})$ and appropriate channels.

\begin{tabular}{llll}
\hline channel & Polarization & output signal & horn \\
\hline a/e & LCP & TP A & M/W \\
b/f & RCP & TP B & M/W \\
c/g & $\operatorname{cross}(\mathrm{Q})$ & $\sin (\mathrm{A} / \mathrm{B})$ & $\mathrm{M} / \mathrm{W}$ \\
$\mathrm{d} / \mathrm{h}$ & $\operatorname{cross}(\mathrm{U})$ & $\cos (\mathrm{A} / \mathrm{B})$ & $\mathrm{M} / \mathrm{W}$ \\
\hline
\end{tabular}

Within the Müller matrix the element $m_{11}$ and accordingly the "sensitivity" $m_{11}^{-1}$ calibrate the total intensity while the $2 \times 2$ matrix with the elements $m_{22}, m_{23}, m_{32}, m_{33}$ translates between biased and unbiased Stokes parameters containing linear polarization information. Here we use the vector $(I, Q, U)_{\mathrm{p}}^{\mathrm{T}}$ that is the vector $\mathbf{S}_{\mathrm{src}}$ already rotated with the parallactic angle of the observation. The expansion of Eq. 2 yields a system of three linear equations. As we observe a set of multiple calibrators of known polarization several times each, each of the three linear equations again forms a system of equations with three matrix elements as unknown parameters. In our case these three equation systems are overdetermined and a least-squared fit can be used to determine the unknown parameters. Due to the linearity of the equations the fit can be solved directly.

\section{Realization with the Effelsberg 100-m Radio Telescope}

In the F-GAMMA program, observations at 11 frequencies between 2.7 and $43 \mathrm{GHz}$ are being conducted. Here, we focus on the analysis of the data sets at 5 and $10 \mathrm{GHz}$. The receivers at these two frequencies show similar performance. The Effelsberg 100-m Telescope provides an overall system with high sensitivity and gain.

\subsection{The Setup}

Table 1 gives an overview of the input and the output of the used receivers fed both by the main horn (denoted as "M") that is pointed on source and the weather horn ("W") that is pointed slightly off source to measure the nearby background. For the calibration of linearly polarized channels, data from the weather horn can be neglected as any influence of it cancels out. Each horn is recording both left- and right handed circular polarized signals (LCP/RCP) entering the receiver system. The signals are getting auto-correlated yielding the direct total power (TP) output signals TP A and B combining to total intensity as TPA + TPB. Furthermore, both signals additionally pass the polarimeter calculating cross terms equaling the Stokes parameters $Q$ and $U$.

\subsection{Calibration of data from the F-GAMMA program}

The observing mode used by the F-GAMMA program is based on "cross-scans", that is, slewing over the source position both in azimuth and elevation direction. Some non-variable and well-studied $[15,16]$ sources $^{1}$ within the sample are required to calibrate the data. We use 3C 286, 3C 161, 3C 48 as linearly polarized sources and NGC 7027

\footnotetext{
${ }^{1}$ http://asa.usno.navy.mil/
}

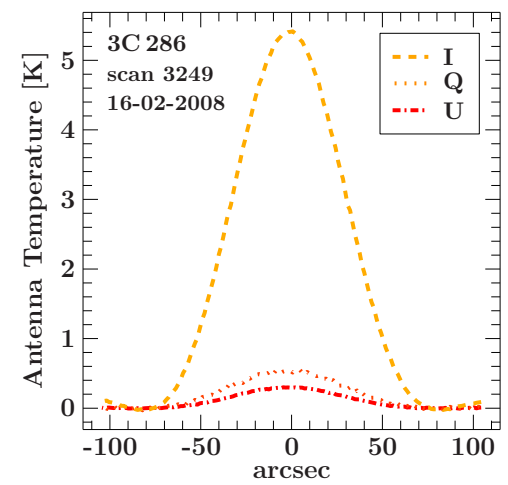

Figure 1. Effelsberg cross-scan profiles for the polarization calibrator $3 \mathrm{C} 286$ at $10 \mathrm{GHz}$ in total intensity, $\mathrm{Q}$ and $\mathrm{U}$ equaling the channels TP A + TP B, $\sin (\mathrm{A} / \mathrm{B})$ and $\cos (\mathrm{A} / \mathrm{B})$.

as unpolarized one. Typically, 2 to 4 calibrators are observed several times in each F-GAMMA observing session. Given the often small number of observed calibrators, it is challenging to compute the correct Müller matrix representing the receiver system that is supposed to be relatively stable. Figure 1 shows the raw data of a single scan on the polarization calibrator $3 \mathrm{C} 286$ at $10 \mathrm{GHz}$. The cross-scans of Stokes Q and U are about an order of magnitude weaker but still of high enough signal-to-noise. The average PD of 3C 286 is $11.7 \%$ at $10 \mathrm{GHz}$ and $11 \%$ at $5 \mathrm{GHz}$ which makes it a very important and valuable calibrator. After summer 2010 the method of extracting the Stokes parameters Q and U from cross-scans changed. Previously they were extracted as amplitudes from Gaussian fits to the appropriate profiles. Beam-squint effects, however, lead to different beam shapes for the channels delivering Stokes Q and U [6]. The total intensity beam can be expected to be centered on the source position. Thus total intensity scans are used to measure the source position in azimuth and elevation. The Stokes Q and U values can now be read off at that position from their scan-profiles. In addition, the uncertainty of Stokes $Q$ and $U$ values is no longer derived from uncertainties of the Gaussian fit parameters underestimating the "true" ones but as rms from the scatter of Stokes Q and U values around their profile maximum. The more conservative uncertainties improve the statistics of the Müller matrix fitting. Having derived the raw-data from cross-scans, one Müller matrix for each observing session is fitted based on raw-data of observed calibrator sources. Appropriate calibration matrices are then applied to the those of the observed calibrators. The results should yield Stokes parameters that are free of instrumental bias and ideally equal the input values. In practice the results follow a Gaussian-like distribution as it is shown in Fig. 2 for $3 \mathrm{C} 286$ at $10 \mathrm{GHz}$ for total intensity, PD and the EVPA. The width of the broadest Gaussian of one parameter and all calibrators derived via least-square fits is used as repeatability uncertainty in addition to the statistical uncertainties of the calibrated parameters. The latter equal the uncertainties of observed Stokes parameters propagated via the inverse Müller matrix.

\subsection{Examination of the derived Müller matrices}

A calibration using Müller matrices naturally includes the determination of the sensitivity $m_{11}^{-1}$ expressed in 

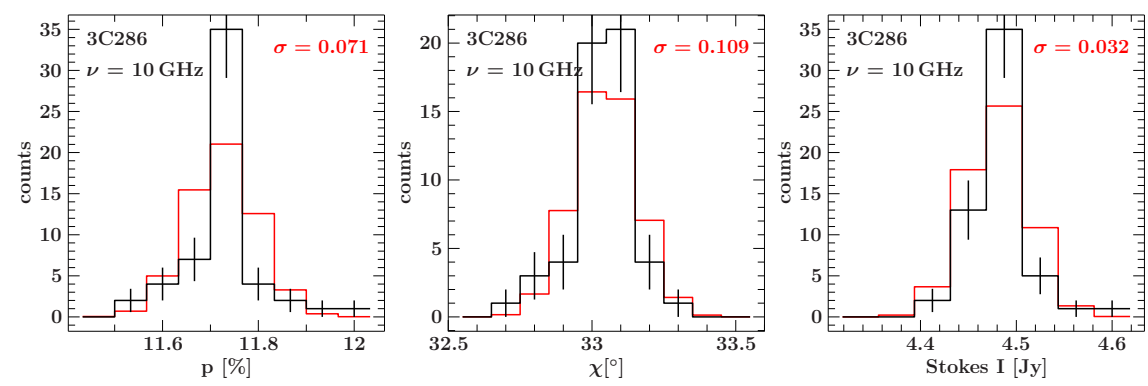

Figure 2. Distribution of

calibrated data in total intensity, PD and the EVPA for the calibrator 3C 286 at $10 \mathrm{GHz}$. Black lines form the histogram that is fitted with a Gaussian in red.
Table 2. Average sensitivity factors 5 and $10 \mathrm{GHz}$ receivers.

\begin{tabular}{lll}
\hline & $m_{11}$ & $m_{11}^{-1}$ \\
\hline $28 \mathrm{~mm}$ & $0.78 \pm 0.06$ & $1.26 \pm 0.56$ \\
$60 \mathrm{~mm}$ & $0.66 \pm 0.04$ & $1.52 \pm 0.05$ \\
\hline
\end{tabular}

units of $[\mathrm{K} / \mathrm{Jy}]$. The sensitivities remain relatively constant over time. Table 2 summarizes the derived average sensitivity factors with standard deviations. The temporal behavior of the other Müller matrix elements are encoded in quantities as the instrumental polarization $p_{\text {instr }}=\sqrt{m_{21}^{2}+m_{31}^{2}} /\left|m_{11}\right|$, the instrumentally induced EVPA $\chi_{\text {instr }}=\arctan \left(m_{31} / m_{21}\right)-$ while any other EVPA rotation is contained in the lower right $2 \times 2$ rotation matrix with the angle $\chi_{\text {rot }}=\arctan \left[\left(m_{32}-m_{23}\right) /\left(m_{22}+m_{33}\right)\right]$ - and the depolarization term $p_{\text {depol }}=\left(m_{12}^{2}+m_{13}^{2}\right) /\left|m_{11}\right|$. We do not further discuss the values of depolarization as they are hard to derive and thus underlie strong scatter. The instrumentally induced polarization stays constant over the full time of monitoring in the range of $0-1 \%$ at $5 \mathrm{GHz}$. At $10 \mathrm{GHz}$ we find similar values until the beginning of 2011 with a sudden increase to $\sim 9 \%$. The parameters $\chi_{\text {instr }}$ and $\chi_{\text {rot }}$ at $5 \mathrm{GHz}$ are subject to some scatter reaching values of $-40^{\circ}<\chi_{\text {instr }}<-80^{\circ}$ and $-100^{\circ}<\chi_{\text {rot }}<150^{\circ}$ before summer 2010. Afterwards we find mean values of $\chi_{\text {instr }} \sim-50^{\circ}$ and $\chi_{\text {rot }} \sim 90^{\circ}$. At $10 \mathrm{GHz}$ the scatter reaches up to summer 2011. We find values of $-90^{\circ}<\chi_{\text {instr }}<90^{\circ}$ and $-140^{\circ}<\chi_{\text {rot }}<140^{\circ}$. Both converge to $\chi_{\text {instr }} \sim-40^{\circ}$ and $\chi_{\text {rot }} \sim 0^{\circ}$ after summer 2011.

The reason for the convergence right after the change of the reduction pipeline in summer 2010 is the use of more conservative uncertainties for the cross-scan derived parameters Stokes Q and U. That allows the Müller matrix fits to converge to one similar solution. The delayed convergence at $10 \mathrm{GHz}$ is due to too large uncertainties of measured Stokes Q and U values up to summer 2011 manifesting themselves in widely differing Müller matrix elements. After summer 2011 the phase switch within the $10 \mathrm{GHz}$ receiver got broken. At the same time the uncertainties start to be more realistic making $\chi_{\text {instr }}$ and $\chi_{\text {rot }}$ converge, which may be a coincidence. The switch allows to subtract two signals of $180^{\circ}$ phase difference within the polarimeter reducing the noise. The software, however, can still extract polarimetry data from these epochs ignoring the phase switch. The rise of the instrumental PD might be connected with that. At the cost of matrices be-
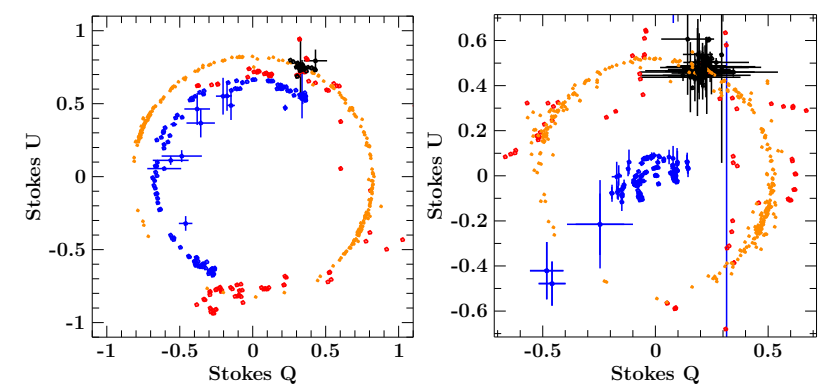

Figure 3. Multi-epoch Q-U plots for 3C 286 at $5 \mathrm{GHz}$ (left) and $10 \mathrm{GHz}$ (right). Red and blue data points correspond to observed data before and after summer 2010, black points the calibrated data and orange points calibrated data rotated with appropriate parallactic angles.

ing variable in that respect, the overall calibration is stable within the uncertainties.

\subsection{Results of calibration}

The results of the calibration are best demonstrated in Fig. 3. Here the complete raw data of 3C 286 are plotted in red when originating from epochs before summer 2010 and in blue for epochs after that. Black represents data calibrated with the derived Müller matrices scattering within a point-cloud distribution. Those points are additionally rotated when applying a rotation matrix with the appropriate parallactic angle of the respective observation covering a part of a circle (orange points). In absence of an instrumental bias the parallactic rotation of the EVPA would result in those brown data distributed on a circle centered around zero with the polarized intensity equal to the radius (Fig. 3). Obviously the raw data are deviating from that circle. The radius is reduced by the sensitivity calibration factors $m_{22}$ and $m_{33}$ while the orientation is changed by any rotation induced by the $2 \times 2$ sub-matrix described by the elements $m_{22}, m_{23}, m_{32}, m_{33}$.

\section{Long-term polarimetry of $3 \mathrm{C} 111$}

The influence of extended emission on integrated linear polarization measurements has to be fully understood before interpreting calibrated polarimetry data. Most FGAMMA program sources are blazars, which do not show substantial extended emission resolved by the Effelsberg beam. In the more complex case of the radio galaxy 3C 111, we mapped the kiloparsec scale structure at both $5 \mathrm{GHz}$ and $10 \mathrm{GHz}$. At $5 \mathrm{GHz}$, the $\sim 200$ arcsec scale structure [11] is nearly unresolved. At $10 \mathrm{GHz}$ (Fig. 5), the two lobes are clearly resolved from the core emission. Crossscans are driven along the indicated arrows in Fig. 5 and are picking up the core and inner jet emission at $10 \mathrm{GHz}$ 


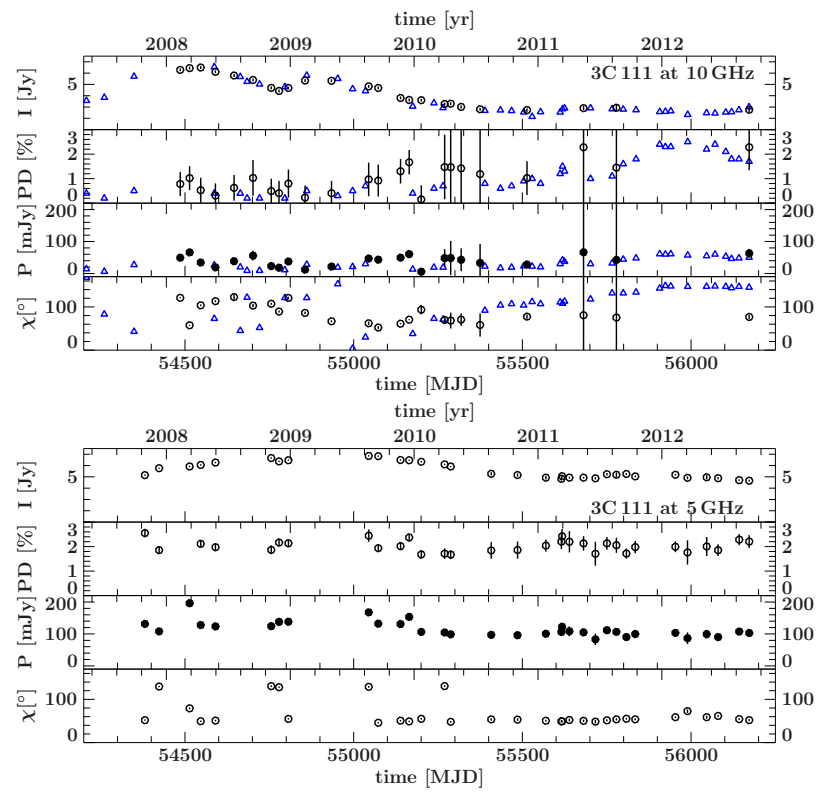

Figure 4. Polarimetry light curve of 3C 111 at $10 \mathrm{GHz}$ (top) and at $5 \mathrm{GHz}$ (bottom). See the text for more details.

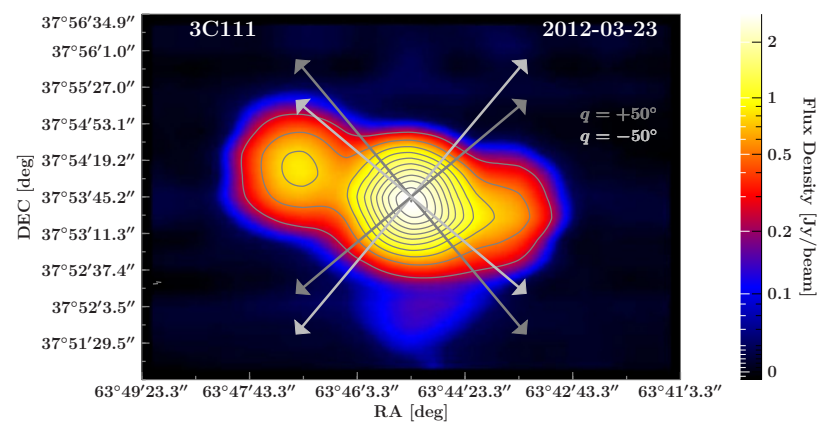

Figure 5. Effelsberg $10 \mathrm{GHz}$ map of the linear polarized flux density $P$ from 23-03-2012. We use B1950.0 coordinates. Contour levels are $(0,13,27,41,55,69,82) \mathrm{mJy}_{\text {beam }}{ }^{-1}$. Arrows denote cross-scan az/elv driving directions for the two parallactic angles $q=+50^{\circ}$ (dark gray) and $-50^{\circ}$ (light gray) under which $3 \mathrm{C} 111$ has been observed.

only. The light curves of I, PD, P, and EVPA (Fig. 4) show pronounced variability in contrast to the ones at $5 \mathrm{GHz}$. With help of simultaneous MOJAVE ${ }^{2}$ VLBI images we interpret this as a signature of core and inner jet variability due to ejecta possibly combined with shocks that may locally align the magnetic field. That would result in an enhanced degree of polarization [8, 9, 17-20]. Integrated values from the MOJAVE monitoring (blue triangles, Fig. 4) are in good agreement with the light curves at $10 \mathrm{GHz}$. The PD-maximum at $10 \mathrm{GHz}$ in early 2012 can be attributed to a VLBI jet component ejected in 2009 trailing behind and interacting with a leading component in 2012 [7].

\section{Conclusions}

For the first time we study the long term stability of polarimetry calibration for the 5 and $10 \mathrm{GHz}$ receivers of the 100-m Effelsberg Radio Telescope. We derive one Müller matrix for each observing session to calibrate the polarimetric raw data with the by-product of total intensity calibration perfectly consistent with former work. It appears essential to properly estimate the uncertainties on measured raw data when calibrating weak signals in polarized channels with a limited set of calibrator observations. Especially, the $2 \times 2$ matrix elements $m_{22}, m_{23}, m_{32}, m_{33}$ and the derived parameters describing instrumental rotations and cross-term effects between Stokes Q and U is very sensitive to calibration. The stable calibration is ensured by some variability of calibration matrices not being due to unstable receivers. We also analyze Effelsberg calibrated data of $3 \mathrm{C} 111$ at $10 \mathrm{GHz}$ revealing pronounced variability due to shocked jet components identified as well in high resolution MOJAVE VLBI maps.

\section{References}

[1] Aller, H. D., Aller, M. F., \& Hodge, P. E. 1981, AJ, 86,325

[2] Aller, H. D., Aller, M. F., \& Hughes, P. A. 1985, ApJ, 298, 296

[3] Angelakis, E., Fuhrmann, L., Nestoras, I., et al. 2010, arXiv: 1006.5610

[4] Antonucci, R. 2012, Astronomical and Astrophysical Transactions, 27, 557

[5] Fanaroff, B. L., \& Riley, J. M. 1974, MNRAS, 167, 31

[6] Fiebig, D., Wohlleben, R., Prata, A., \& Rusch, W. V. T. 1991, IEEE Transactions on Antennas and Propagation, 39, 774

[7] Grossberger, C., Kadler, M., Wilms, J., et al. 2012, Acta Polytechnica, 52, 18

[8] Kadler, M., Ros, E., Perucho, M., et al. 2008, ApJ, 680,867

[9] Laing, R. A. 1980, MNRAS, 193, 439

[10] Legg, M. P. C., \& Westfold, K. C. 1968, ApJ, 154, 499

[11] Linfield, R., \& Perley, R. 1984, ApJ, 279, 60

[12] Lister, M. L., \& Homan, D. C. 2005, AJ, 130, 1389

[13] Saikia, D. J., \& Salter, C. J. 1988, Ann. Rev. Astron. Astrophys., 26, 93

[14] Savolainen, T., Ros, E., Porcas, R. W., \& Zensus, J. A. 2010, Proc. "Fermi meets Jansky - AGN in Radio and Gamma Rays", MPIfR, p.81-84, 2010

[15] Tabara, H., \& Inoue, M. 1980, A\&AS, 39, 379

[16] Turlo, Z., Forkert, T., Sieber, W., \& Wilson, W. 1985, A\&A, 142, 181

[17] Marscher, A. P., \& Gear, W. K. 1985, ApJ, 298, 114

[18] Marscher, A. P. 1996, Energy Transport in Radio Galaxies and Quasars, 100, 45

[19] Marscher, A. P. 2006, Relativistic Jets: The Common Physics of AGN, Microquasars, and Gamma-Ray Bursts, 856, 1

[20] Wardle, J. F. C., Cawthorne, T. V., Roberts, D. H., \& Brown, L. F. 1994, ApJ, 437, 122

${ }^{2}$ http://www.physics.purdue.edu/astro/MOJAVE/ 